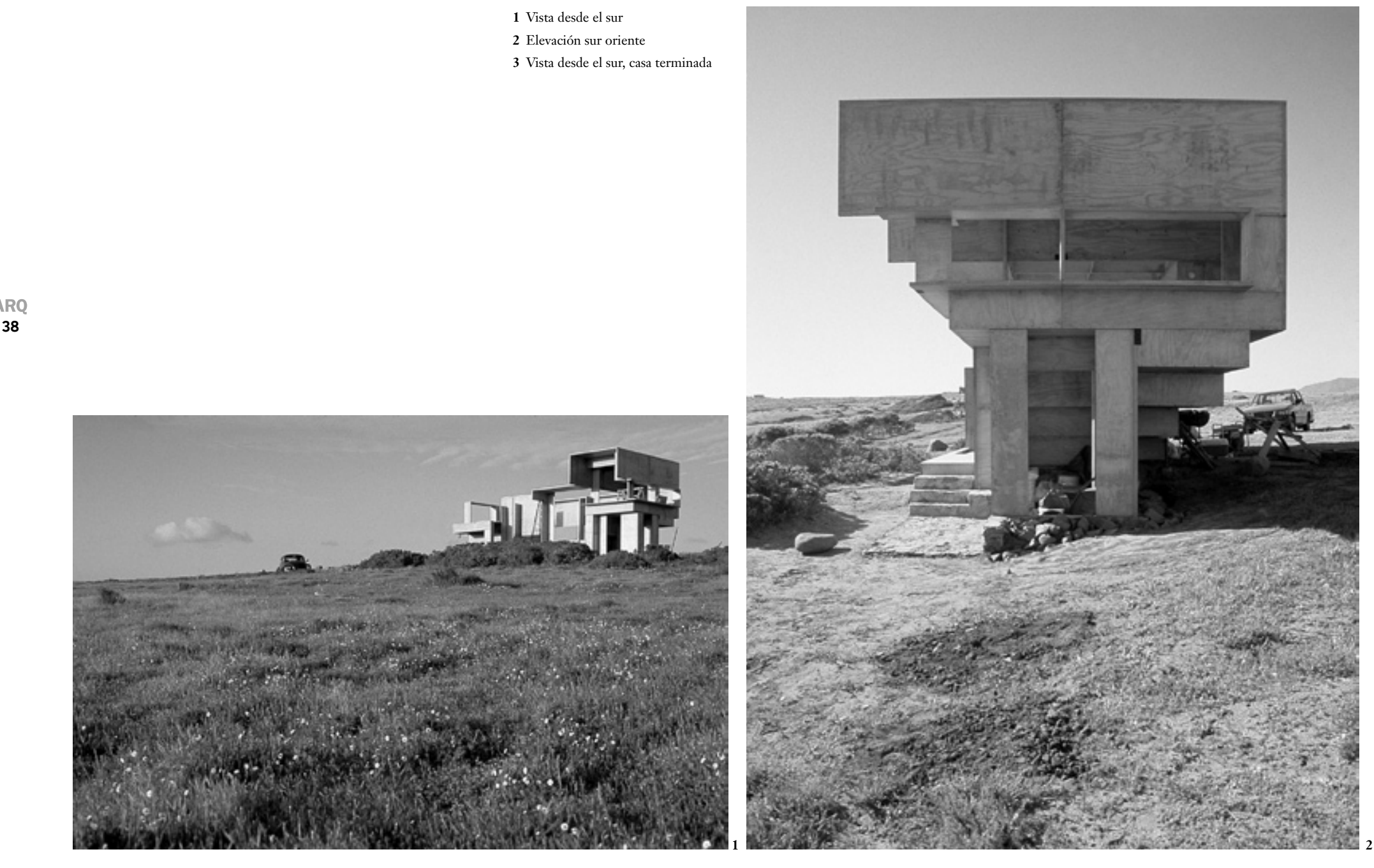

Cooperativa URO1.ORG

Tunquén, Chite

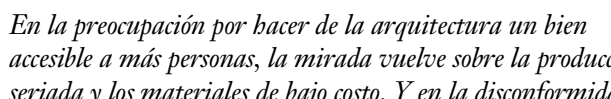

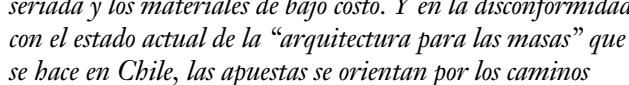

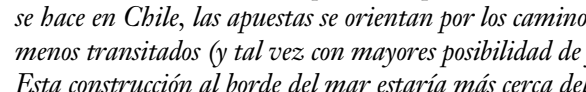

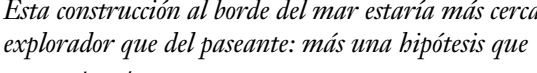

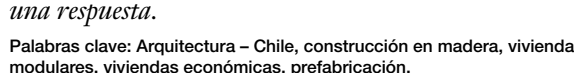

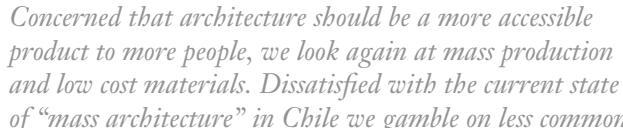

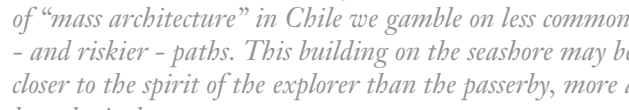

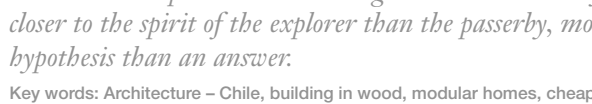
dispuestos a asumir los riesgos (y costos) que descontar de sus costos algo así como el $7 \%$ contemporíneas de la economín las regla

\section{Prototipo M7}

Desde que la arquitectura se inscribe en lo $q$ puede entenderse como aquello que posee Este escenario deja a la mayoría de la ha derivado exploración estética, cuando a los beneficios de una costos an pritadectus ésta propicia un avance en el estado del arte, o en experimentación técnica o funcional en edificación, cuando ésta logra situarse como

M7, edición con errores. El artefacto casa En nuestro medio, un problema central es
dificultad para encontrar clientes que este conlleva el desarrollo de estas exploraciones
arquitectónicas. Cuando la arquitectura moderna asume, por ejemplo, el proyecto de la vivienda económica, no sólo daba un gran paso al incluir
otro tipo edificatorio dentro de lo que hasta es entonces era llamado "arquitectura"; adem y pruaba al Estado como entidad que asumm
y promovgo que estas experiencias implicaban. Hoy resulta difíili que el Estado-
nación en creciente crisis, asuma los riesgos una arquitectura artesanal que pocos quieren
costear, y que sólo a veces es financiada costear, y que solo a veces es financiada
una elite que actúa como mecenas de autoresdemas dificulta el trabajo de los arquitecto desplazados por miembros de otras disciplinas infraestructuras convencionales, y que puede arquitecto -si no más, considerando los gastos asumidos indirectamente por arquitectos,

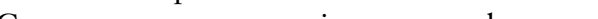
mercado, o bien se entiende que la arquitectura es un valor agregado costoso y deseable por prober andiza el costo de diseñ en la industria de vestuario ${ }^{4}$. Podemos considerar el acceso amplio y masivo a ciertos bienes de consumo arquitectónicos como fenómeno democratizador?, éla aparición tutodetermincín propin de b viduda a
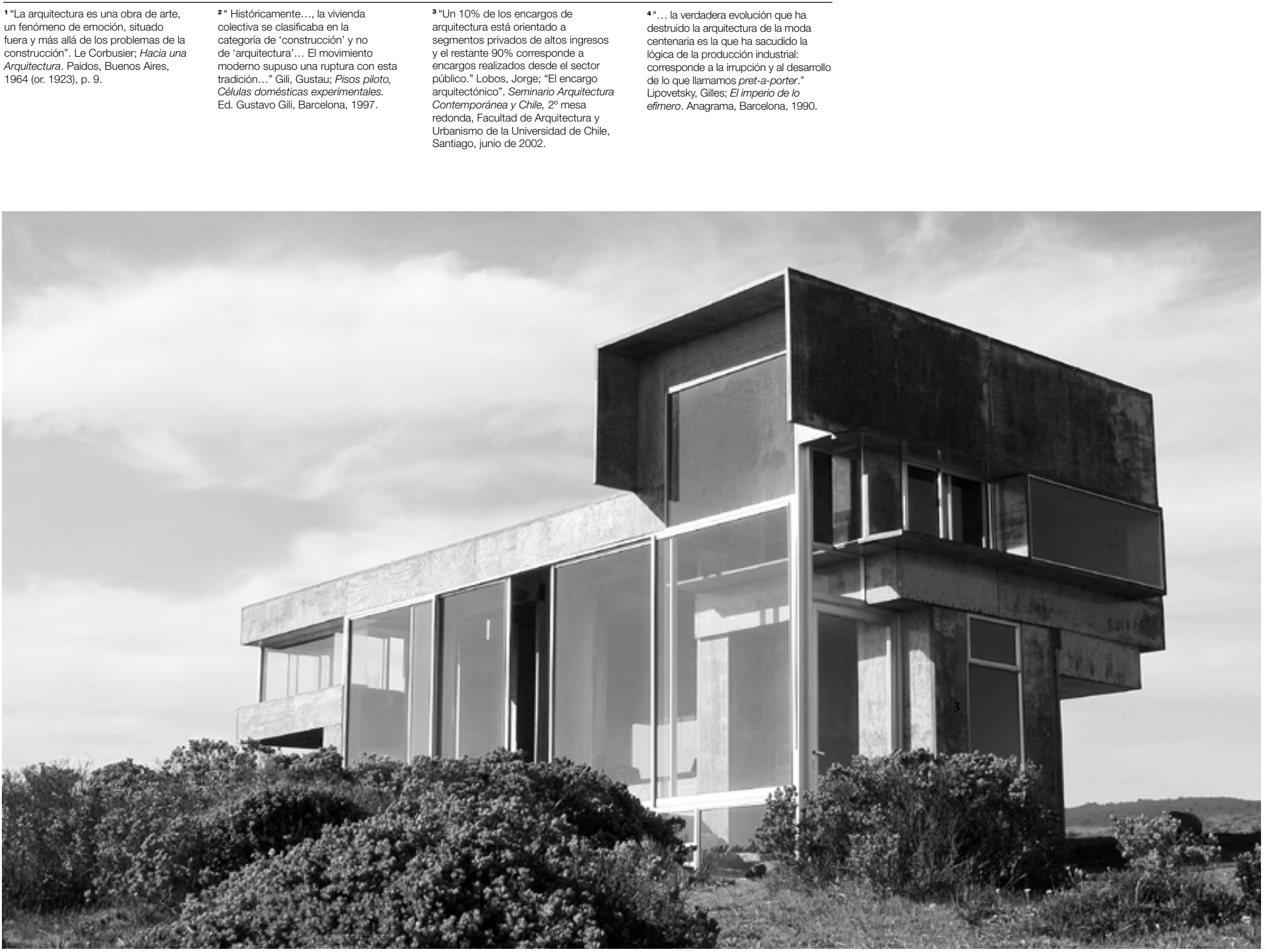
artistitios, procurando desarrollar un producto que $M 7$ como primer prototipo de un nuevo sistema como ocurre en la arquitectura artesanal
Hemos decidido aproximarnos a la idea de
utilizacion de berramientas de mercado con fines

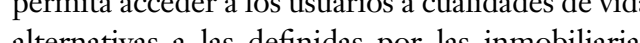
tradicionales. Este es el trance en que se encuentra edificatorio para viviend

$\begin{array}{ll}\text { madera terciada como materia prima, considerando } & \text { como elementos estructurales del tipo viga o } \\ \text { su calidad como producto de mercado (obedece } & \text { contrafuerte. La medida de estos desniveles ( } 400\end{array}$ importantes normas internacionales de $\delta 800 \mathrm{~mm}$ ) es relativamente versatil coincidiendo

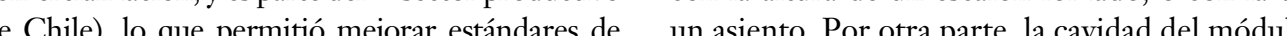
producción y de terminación. Los tableros terciados usados son de pino radiata, especíico dependiendo del espacio al que esté $1220 \times 2440 \times 21 \mathrm{~mm}$; el tablero final con el que enmarca en una estrategia de alejamiento de como hobby aristocrático, producto del sistema de elite en que nos educamos como arquitectos. Nos interesa indagar sobre $\mathrm{d}$ poder de decisión de los

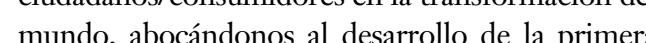
etapa de un producto arquitectónico que por su relación costo/calidad, pueda ser accesible para un rango más amplio de población. $M 7$ es un habitaculo originado por la utilizacion de una piezzá
estandar pre-armada que, a pesar de su condición, posibilita variaciones formales $y$ espaciales acuerdo a los requerimientos de cada cliente.

27 piezas ce tercindo $y$ el optimizando el uso del formato de tercindo ctúa indiferentemente como muro, suelo o tech según su posición horizontal o vertical, variando su
conformación según las necesidades estructurales conformación según las necesidades estructurales

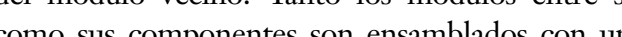
mismo tipo de unión; una vez montados, los 27 módulos de la casa se comports pieza estructural monomaterial a administración de este módulo permi planos constructivos, que al mismo tiempo act
La superficie lisa que queda expuesta a capa de resina acrílica tras flexible y filtro UV de un espesor promedio de Resultado

a experiencia del prototipo $M 7$ respondió tisfactoriamente a las premisas planteadas: 1.El costo por metro cuadrado fue de $12 \mathrm{UF}$. equipo de 6 personas. sus aciertos y errores, están siendo procesadas para mercado inmobitario a proceso de transforma obra gruesa terminada, se idearon módulos de 2400 menos de media plancha de terciado. 


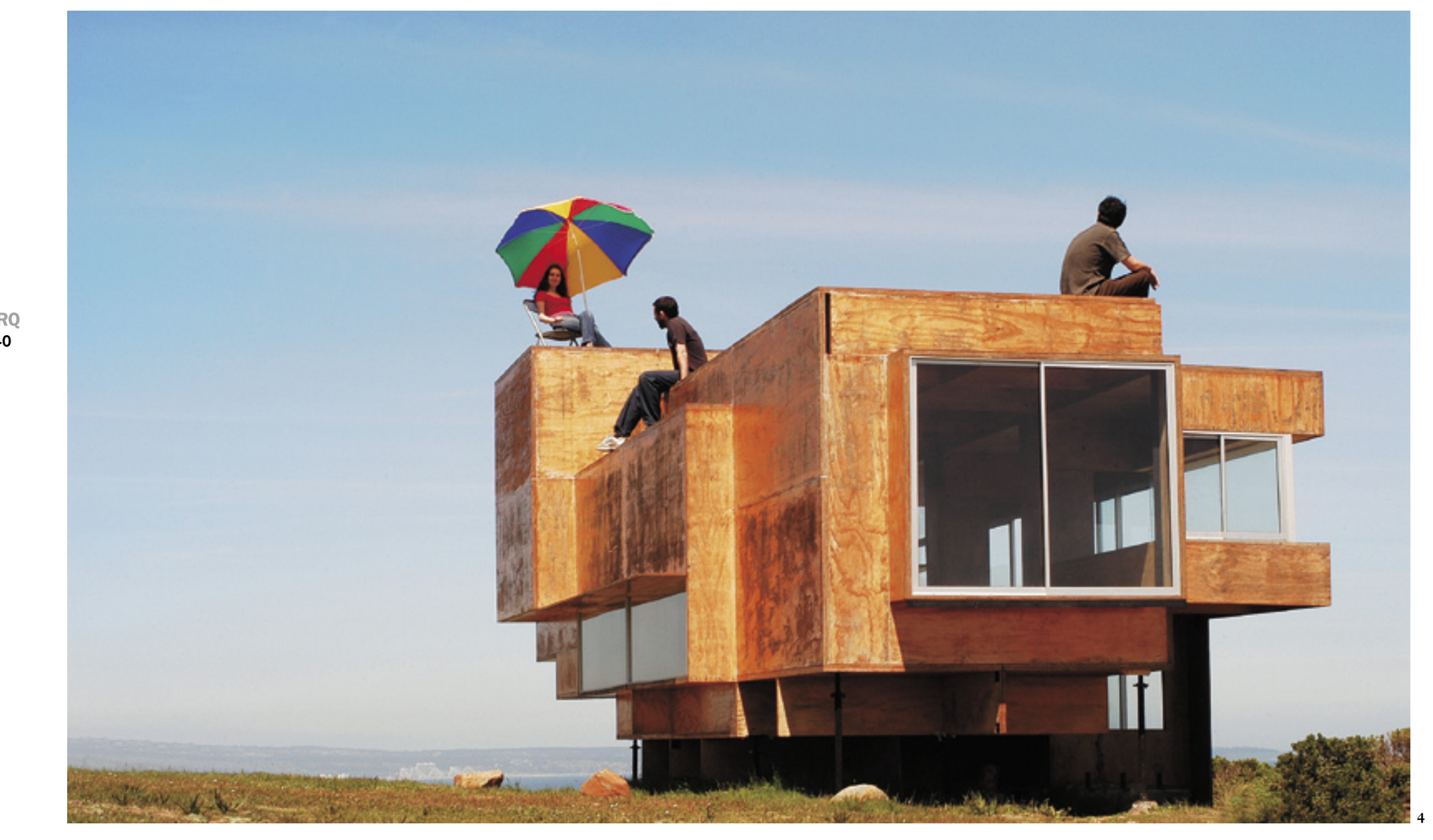

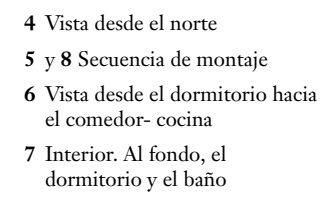
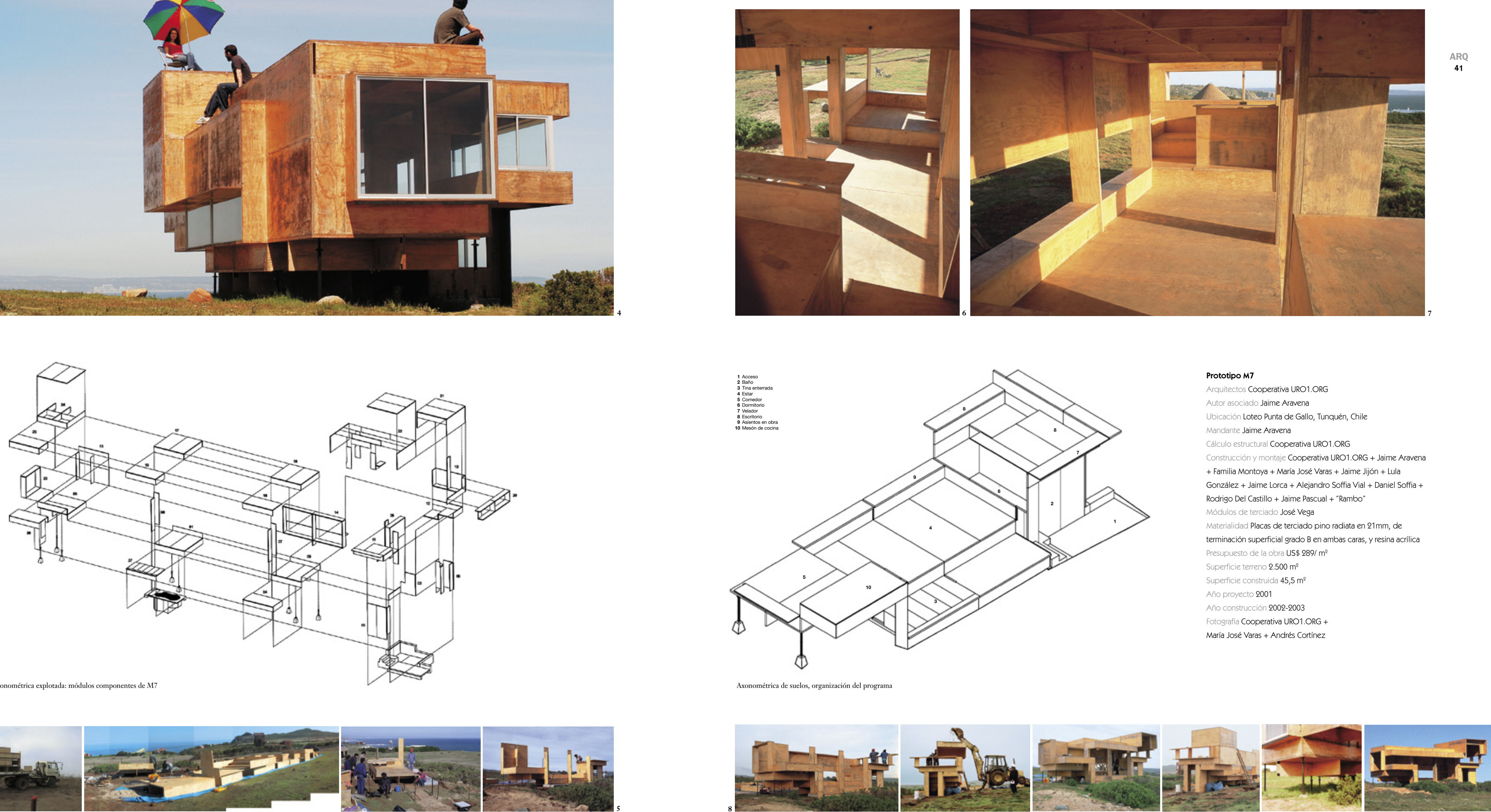

$\underset{41}{4 R Q}$

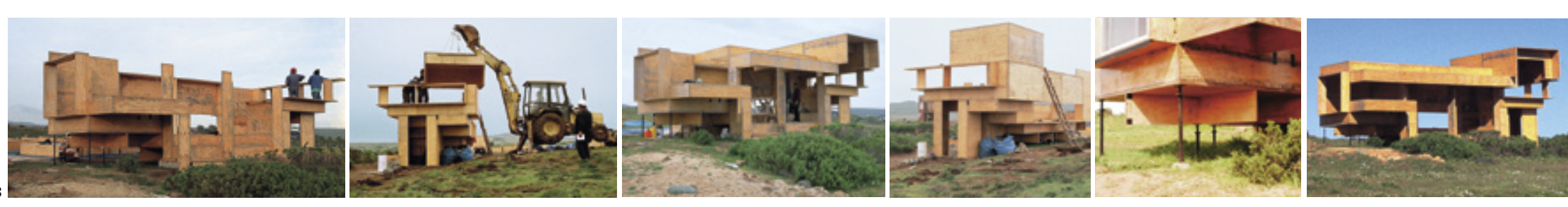

